\title{
Learning from Imprecise Data: Possibilistic Graphical Models
}

\author{
Christian Borgelt and Rudolf Kruse \\ Dept. of Knowledge Processing and Language Engineering \\ School of Computer Science \\ Otto-von-Guericke-University of Magdeburg \\ Universitätsplatz 2, D-39106 Magdeburg, Germany
}

\begin{abstract}
Graphical models - especially probabilistic networks like Bayes networks and Markov networks - are very popular to make reasoning in highdimensional domains feasible. Since constructing them manually can be tedious and time consuming, a large part of recent research has been devoted to learning them from data. However, if the dataset to learn from contains imprecise information in the form of sets of alternatives instead of precise values, this learning task can pose unpleasant problems. In this paper we study an approach to cope with these problems, which is not based on probability theory as the more common approaches like, e.g., expectation maximization, but uses possibility theory as the underlying calculus of a graphical model.
\end{abstract}

Keywords: graphical models, possibilistic networks, learning from data

\section{Introduction}

Since reasoning in high-dimensional domains tends to be infeasible in the domains as a whole and the more so if uncertainty and imprecision are involved-decomposition techniques, which reduce the reasoning process to computations in lower-dimensional subspaces, have become very popular. Decomposition based on independence relations between variables, for example, has been studied extensively in the field of graphical modeling, in which graphs (in the sense of graph theory) are used to describe decompositions of distributions. Among the best-known approaches are Bayes networks [Pearl 1988], Markov networks [Lauritzen and Spiegelhalter 1988], and the more general valuation-based networks [Shenoy 1992]. All of these approaches led to efficient implementations, for example HUGIN [Andersen et al. 1989], PULCINELLA [Saffiotti and Umkehrer 1991], and PATHFINDER [Heckerman 1991].

Due to the fact that a graphical model is a comprehensive description of the dependences and independences that hold in a given domain and allows us to draw inferences efficiently, it is a powerful tool to support reasoning - as soon as it is constructed. Its construction by human experts, however, can be tedious and time consuming. Therefore recent research in graphical models focused on methods to learn them from a database of sample cases. Although 
some important instances of this learning task have been shown to be NPhard in the general case [Dechter and Pearl 1992, Chickering et al. 1994], there are several highly successful heuristic algorithms [Cooper and Herskovits 1992, Heckerman et al. 1995, Gebhardt and Kruse 1995, Jordan 1998].

Several of these approaches, however, are restricted to learning from precise data, i.e., the description of the sample cases must not contain missing values or set-valued information: There must be exactly one value for each of the attributes used to describe the domain. In applications this presupposition is rarely met, though: Databases are notoriously incomplete and useful imprecise information (in the sense of a set of values for an attribute) is frequently available (although it is often discarded, because standard database implementations cannot handle it adequately). Hence we are faced with the challenge to extend the existing learning algorithms to incomplete and imprecise data.

In the field of probabilistic graphical models it is tried to meet this challenge with approaches that are based on the expectation maximization (EM) algorithm [Dempster et al. 1977, Bauer et al. 1997, Friedman 1998]. Although these approaches are promising, we explore a different path in this paper, namely graphical models that are based on possibility theory [Gebhardt 1997, Borgelt 2000]. It turns out that with this type of graphical models imprecise information can be handled very conveniently.

\section{Graphical Models}

The basic idea underlying graphical models is to exploit independence relations between variables in order to decompose a high-dimensional distributioni.e., a relation, a probability distribution, or a possibility distribution - into a set of (conditional or marginal) distributions on lower-dimensional subspaces. This decomposition - and the independence relations that make it possibleis represented as a (directed or undirected) graph: There is a node for each attribute used to describe the considered domain. Edges connect attributes that are directly dependent on each other. They also indicate the paths along which evidence has to be propagated, when inferences are to be drawn.

\subsection{Decomposition}

The notion of decomposition is perhaps best known from the theory of relational databases [Ullman 1988] and actually relational database theory is closely connected to the theory of graphical models. The connection is brought about by the notion of the join-decomposability of a relation, which in relational databases is exploited to store a high-dimensional relation with less redundancy and, of course, using less storage space.

The idea underlying join-decomposability is that often a relation can be reconstructed from certain projections of it by forming their so-called natural join. Formally, this can be described as follows: Let $U=\left\{A_{1}, \ldots, A_{n}\right\}$ be a set 
of attributes and let $\operatorname{dom}\left(A_{i}\right)$ be their respective domains. Furthermore, let $r_{U}$ be a relation over $U$. We represent this relation by its indicator function, which assigns a value of 1 to all tuples contained in the relation and a value of 0 to all tuples not contained in it. The tuples themselves we represent as conjunctions $\bigwedge_{A_{i} \in U} A_{i}=a_{i}$, which state a value for each of the attributes.

Using an indicator function a projection $r_{M}$ of the relation $r_{U}$ to a subset $M$ of the attributes in $U$ can easily be defined by

$$
r_{M}\left(\bigwedge_{A_{i} \in M} A_{i}=a_{i}\right)=\max _{\substack{\forall A_{j} \in U-M: \\ a_{i} \in \operatorname{dom}\left(A_{j}\right)}} r_{U}\left(\bigwedge_{A_{i} \in U} A_{i}=a_{i}\right),
$$

where the somewhat sloppy notation w.r.t. the maximum is meant to indicate that the maximum has to be taken over all values of all attributes in $U-M$. With this notation a relation $r_{U}$ is called join-decomposable w.r.t. a family $\mathcal{M}=\left\{M_{1}, \ldots, M_{m}\right\}$ of subsets of $U$ iff

$$
\begin{aligned}
& \forall a_{1} \in \operatorname{dom}\left(A_{1}\right): \ldots \forall a_{n} \in \operatorname{dom}\left(A_{n}\right): \\
& \quad r_{U}\left(\bigwedge_{A_{i} \in U} A_{i}=a_{i}\right)=\min _{M \in \mathcal{M}} r_{M}\left(\bigwedge_{A_{i} \in M} A_{i}=a_{i}\right) .
\end{aligned}
$$

Note that the minimum operation used here is equivalent to the natural join of relational algebra. It is obvious that in such a situation it suffices to store the projections $r_{M}$ in order to capture all information contained in the relation $r_{U}$, because we can always reconstruct the original relation.

The decomposition scheme we just outlined for the relational case is easily transferred to the probabilistic case: We only have to replace the projection and the natural join by the proper probabilistic operations. Thus we arrive at

$$
\begin{aligned}
& \forall a_{1} \in \operatorname{dom}\left(A_{1}\right): \ldots \forall a_{n} \in \operatorname{dom}\left(A_{n}\right): \\
& \quad p_{U}\left(\bigwedge_{A_{i} \in U} A_{i}=a_{i}\right)=\prod_{M \in \mathcal{M}} \phi_{M}\left(\bigwedge_{A_{i} \in M} A_{i}=a_{i}\right) .
\end{aligned}
$$

The functions $\phi_{M}$ can be computed from the marginal distributions on the sets $M$ of attributes, which shows that marginalization takes the place of projection. These functions are called factor potentials [Castillo et al. 1997].

The possibilistic case is even closer to the relational one, since the decomposition formula is identical:

$$
\begin{aligned}
& \forall a_{1} \in \operatorname{dom}\left(A_{1}\right): \ldots \forall a_{n} \in \operatorname{dom}\left(A_{n}\right): \\
& \quad \pi_{U}\left(\bigwedge_{A_{i} \in U} A_{i}=a_{i}\right)=\min _{M \in \mathcal{M}} \pi_{M}\left(\bigwedge_{A_{i} \in M} A_{i}=a_{i}\right) .
\end{aligned}
$$

The only difference is that the possibility distributions $\pi_{U}$ and $\pi_{M}$ are not restricted to the values 0 and 1 as an indicator function is, but can assume any value in the interval $[0,1]$. In this way a gradual possibility of a tuple is modeled and possibilistic graphical models can be developed as "fuzzifications" of relational graphical models. 
For the semantics of such degrees of possibility we rely on the context model [Gebhardt and Kruse 1993]: Suppose that for a description of the modeled domain we can distinguish between a set $C=\left\{c_{1}, \ldots, c_{k}\right\}$ of contexts. These contexts may be given, for example, by physical or observation-related frame conditions. Furthermore, suppose that we can describe the relative importance or frequency of occurrence of these contexts by assigning a probability $P(c)$ to each of them. Finally, suppose that we can state for each context $c$ a set $\Gamma(c)$ of possible states - described by tuples - the modeled domain may be in under the physical or observation-related frame conditions that characterize the context. We assume each set $\Gamma(c)$ to be the most specific correct set-valued specification of the state $t_{0}$ of the modeled domain, which we can give for the context c. By "most specific set-valued specification" we mean that we can guarantee that $\Gamma(c)$ contains $t_{0}$, but that we cannot guarantee that a proper subset of $\Gamma(c)$ contains $t_{0}$. Given these ingredients, we define the degree of possibility that a tuple $t$ describes the actual state $t_{0}$ of the modeled section of the world as the weight (probability) of all contexts in which $t$ is possible.

Formally, the above description results in a random set (i.e., a set-valued random variable) $\Gamma: C \rightarrow 2^{T}$ as an imperfect (i.e., imprecise and uncertain) specification of the actual state $t_{0}$ of the modeled section of the world. From it we derive a possibility distribution by simply computing its one-point coverage

$$
\pi_{\Gamma}: T \rightarrow[0,1], \quad \pi_{\Gamma}(t)=P(\{c \in C \mid t \in \Gamma(c)\}) .
$$

In this interpretation a possibility distribution represents uncertain and imprecise knowledge as can be seen by comparing it to a probability distribution and to a relation. A probability distribution covers uncertain, but precise knowledge. This becomes obvious if one notices that a possibility distribution in the interpretation described above reduces to a probability distribution if $\forall c \in C:|\Gamma(c)|=1$, i.e., if for all contexts the specification of $t_{0}$ is precise. On the other hand, a relation represents imprecise, but certain knowledge. Thus, not surprisingly, a relation can also be seen as a special case of a possibility distribution in the interpretation given above, namely if there is only one context. Hence the context-dependent specifications are responsible for the imprecision, the contexts for the uncertainty.

\subsection{Graphical Representation}

Graphs (in the sense of graph theory) are a very convenient tool to describe decompositions if we identify each attribute with a node. In the first place, graphs can be used to specify the sets $M$ of attributes underlying the decomposition. How this is done depends on whether the graph is directed or undirected. If it is undirected, the sets $M$ are the maximal cliques of the graph, where a clique is a complete subgraph and it is maximal if it is not

contained in another complete subgraph. If the graph is directed, we can be more explicit about the distributions in the decomposition: We can use conditional distributions, since we may use the direction of the edges to specify 
which is the conditioned attribute and which are the conditions. Note, however, that this does not make much of a difference in the relational and the possibilistic case, since here conditional distributions are simple identified with the corresponding marginal distributions, i.e.,

$$
\pi\left(A_{j}=a_{j} \mid \bigwedge_{A_{i} \in M} A_{i}=a_{i}\right)=\pi\left(A_{j}=a_{j} \wedge \bigwedge_{A_{i} \in M} A_{i}=a_{i}\right) .
$$

Secondly, graphs can be used to describe (conditional) dependence and independence relations between attributes via the concept of separation of nodes. What is to be understood by "separation" depends again on whether the graph is directed or undirected. If it is undirected, separation is defined as follows: If $X, Y$, and $Z$ are three disjoint subsets of nodes in an undirected graph, then $Z$ separates $X$ from $Y$ iff after removing the nodes in $Z$ and their associated edges from the graph there is no path, i.e., no sequence of consecutive edges, from a node in $X$ to a node in $Y$. Or, in other words, $Z$ separates $X$ from $Y$ iff all paths from a node in $X$ to a node in $Y$ contain a node in $Z$.

For directed graphs, which have to be acyclic, the so-called $d$-separation criterion is used [Pearl 1988, Verma and Pearl 1990]: If $X, Y$, and $Z$ are three disjoint subsets of nodes, then $Z$ is said to $d$-separate $X$ from $Y$ iff there is no path, i.e., no sequence of consecutive edges (of any directionality), from a node in $X$ to a node in $Y$ along which the following two conditions hold:

1. every node with converging edges either is in $Z$ or has a descendant in $Z$, 2. every other node is not in $Z$.

These separation criteria are used to define conditional independence graphs: A graph is a conditional independence graph w.r.t. a given multi-dimensional distribution if it captures by node separation only correct conditional independences between sets of attributes. Conditional independence means (for three attributes $A, B$, and $C$ with $A$ independent of $C$ given $B$ ) that

$$
P(A=a, B=b, C=c)=P(A=a \mid B=b) \cdot P(C=c \mid B=b)
$$

in the probabilistic case and

$$
\pi(A=a, B=b, C=c)=\min \{\pi(A=a \mid B=b), \pi(C=c \mid B=b)\}
$$

in the possibilistic and the relational case. These formulae demonstrate the close connection of conditional independence and decomposability.

Formally, the connection between conditional independence graphs and graphs that describe decompositions is brought about by theorems that show that a distribution is decomposable w.r.t. a given graph if and only if this graph is a conditional independence graph of the distribution. For the probabilistic setting, this theorem is usually attributed to [Hammersley and Clifford 1971], who proved it for the discrete case, although (according to [Lauritzen 1996]) this result seems to have been discovered in various forms by several authors. In the possibilistic setting similar theorems hold, although certain restrictions have to be introduced [Gebhardt 1997, Borgelt 2000]. 
Finally, the graph underlying a graphical model is very useful to derive evidence propagation algorithms, since evidence propagation can be reduced to simple computations of node processors that communicate by passing messages along the edges of a properly adapted graph. A detailed account can be found, for instance, in [Castillo et al. 1997].

\section{Learning Graphical Models}

Having reviewed the ideas underlying graphical models, we now turn to learning them from a database of sample cases. There are three basic approaches:

- Test whether a distribution is decomposable w.r.t. a given graph. This is the most direct approach. It is not bound to a graphical representation, but can also be carried out w.r.t. other representations of the subsets of attributes to be used to compute the (candidate) decomposition of the given distribution.

- Find an cond. independence graph by conditional independence tests. This approach exploits the theorems mentioned in the preceding section, which connect conditional independence graphs and graphs that describe decompositions. It has the advantage that by a single conditional independence test, if it fails, several candidate graphs can be excluded.

- Find a suitable graph by measuring the strength of dependences.

This is a heuristic, but often highly successful approach, which is based on the frequently valid assumption that in a conditional independence graph an attribute is more strongly dependent on adjacent attributes than on attributes that are not directly connected to it.

Note that none of these methods is perfect. The first approach suffers from the usually huge number of candidate graphs. The second often needs the strong assumption that there is a perfect map (a conditional independence graph that captures all conditional independences by node separation). In addition, if it is not restricted to certain types of graphs (for example, polytrees), one has to test conditional independences of high order (i.e., with a large number of conditioning attributes), which tend to be unreliable unless the amount of data is enormous. The heuristic character of the third approach is obvious. Examples in which it fails can easily be found, since under certain conditions attributes that are not adjacent in a conditional independence graph can exhibit a strong dependence.

A (computationally feasible) analytical method to construct optimal graphical models from a database of sample cases has not been found yet. Therefore an algorithm for learning a graphical model from data usually consists of

1. an evaluation measure (to assess the quality of a given network) and

2. a search method (to traverse the space of possible networks). 
It should be noted, though, that restrictions of the search space introduced by an algorithm and special properties of the evaluation measure used sometimes disguise the fact that a search through the space of possible network structures is carried out. For example, by conditional independence tests all graphs missing certain edges can be excluded without inspecting these graphs explicitly. Greedy approaches try to find good edges or subnetworks and combine them in order to construct an overall model and thus may not appear to be searching. Nevertheless the above characterization is apt, since an algorithm that does not explicitly search the space of possible networks usually carries out a (heuristic) search on a different level, guided by an evaluation measure. For example, some greedy approaches search for the best set of parents of an attribute by measuring the strength of dependence on candidate parent attributes; conditional independence test approaches search the space of all possible conditional independence statements.

\subsection{Computing Projections}

A basic operation needed to learn a graphical model from a dataset of sample cases is a method to estimate from the dataset the marginal or conditional distributions of a candidate decomposition of the universe of discourse. Such an operation is necessary, because the marginal and/or conditional distributions are needed to assess the quality of a given candidate graphical model. We call such an estimation operation a projection, because in the relational setting it corresponds to the projection operation of relational algebra (see above).

If the dataset is precise, i.e., if in all tuples there is exactly one value for each attribute, then computing a projection is trivial in the probabilistic as well as in the possibilistic case, since it consists in counting tuples and computing relative frequencies. However, we consider here learning from imprecise data and this complicates things. As already mentioned above, probabilistic approaches usually rely on some version of the EM algorithm [Dempster et al. 1977] in this case, which is a rather expensive procedure.

In the possibilistic setting it is much simpler to handle imprecise tuples, because with the context model interpretation of a degree of possibility, we have direct means to handle them: We simply interpret each imprecise tuple as a description of the set $\Gamma(c)$ of states of the world that are possible in some context $c$. We can do so, because an imprecise tuple can always be rewritten as a set of tuples, namely the set of all precise tuples compatible with it.

Nevertheless, we face some problems in the possibilistic setting, too, because we can no longer apply naive methods to determine the marginal distributions (a detailed explanation can be found in [Borgelt 2000]). Fortunately, there is a simple preprocessing operation by which the database to learn from can be transformed, so that computing maximum projections becomes trivial [Borgelt and Kruse 1998, Borgelt 2000]. This operation is based on the notion of closure under tuple intersection. That is, we add (possibly imprecise) tuples to the database in order to achieve a situation, in which for any two tuples 
from the database their intersection (i.e., the intersection of the represented sets of precise tuples) is also contained in the database. Details can be found in [Borgelt and Kruse 1998, Borgelt 2000].

\subsection{Evaluation Measures}

An evaluation measure serves to assess the quality of a given candidate graphical model w.r.t. a given database of sample cases, so that it can be determined which of a set of candidate graphical models best fits the given data. A desirable property of an evaluation measure is decomposability, i.e., the total network quality should be computable as an aggregate (e.g. sum or product) of local scores, for example a score for a maximal clique of the graph to be assessed or a score for a single edge.

Most such evaluation measures are based on measures of dependence, since for both the second and the third basic approach listed above it is necessary to measure the strength of dependence of two or more variables, either in order to test for conditional independence or in order to find the strongest dependences. Here we confine ourselves to measures that assess the strength of dependence of two variables in the possibilistic case. The transfer to conditional tests (by computing a weighted sum of the results for the different instantiations of the conditions) and to more than two variables is straightforward.

Possibilistic evaluation measures can be easily derived by exploiting the close connection of possibilistic networks to relational networks (see above). The idea is to draw on the $\alpha$-cut view of a possibility distribution. This concept is transferred from the theory of fuzzy sets [Kruse et al. 1994]. In the $\alpha$-cut view a possibility distribution is seen as a set of relations with one relation for each degree of possibility $\alpha$. The indicator function of such a relation is defined by simply assigning a value of 1 to all tuples for which the degree of possibility is no less than $\alpha$ and a value of 0 to all other tuples. It is easy to see that a possibility distribution is decomposable if and only if each of the $\alpha$-cut relations is decomposable. Thus we may derive a measure for the strength of possibilistic dependence of two variables by integrating a measure for the strength of relational dependence over all degrees of possibility $\alpha$.

To make this clearer, we consider a simple example. Figure 1 shows a simple relation over two attributes $A$ and $B$ : The grey squares indicate the tuples contained in this relation. We can measure the strength of dependence of $A$ and $B$ by computing the Hartley information gain [Hartley 1928]

$$
\begin{aligned}
I_{\text {gain }}^{\text {(Hartley) }}(A, B) & =\log _{2}\left(\sum_{a \in \operatorname{dom}(A)} R(A=a)\right)+\log _{2}\left(\sum_{b \in \operatorname{dom}(B)} R(B=b)\right) \\
& -\log _{2}\left(\sum_{a \in \operatorname{dom}(A)} \sum_{b \in \operatorname{dom}(B)} R(A=a, B=b)\right) \\
& =\log _{2} \frac{\left(\sum_{a \in \operatorname{dom}(A)} R(A=a)\right)\left(\sum_{b \in \operatorname{dom}(B)} R(B=b)\right)}{\sum_{a \in \operatorname{dom}(A)} \sum_{b \in \operatorname{dom}(B)} R(A=a, B=b)} .
\end{aligned}
$$




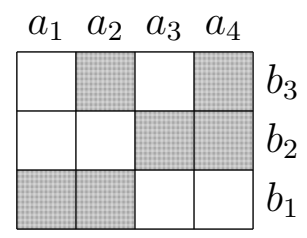

\begin{tabular}{ll} 
Hartley information needed to determine \\
coordinates: & $\log _{2} 4+\log _{2} 3=\log _{2} 12 \approx 3.58$ \\
coordinate pair: & $\log _{2} 6 \quad \approx 2.58$ \\
\hline gain: & $\log _{2} 12-\log _{2} 6=\log _{2} 2=1$
\end{tabular}

Figure 1: Computation of Hartley information gain.

The idea underlying this measure is as follows: Suppose we want to determine the actual values of the two attributes $A$ and $B$. Obviously, there are two possible ways to do this: In the first place, we could determine the value of each attribute separately, thus trying to find the "coordinates" of the value combination. Or we may exploit the fact that the value combination is restricted by the relation shown in figure 1 and try to determine the value combination directly. In the former case we need the Hartley information of the set of values of $A$ plus the Hartley information of the set of values of $B$, i.e., $\log _{2} 4+\log _{2} 3 \approx 3.58$ bits. In the latter case we need the Hartley information of the possible tuples, i.e., $\log _{2} 6 \approx 2.58$ bit, and thus gain one bit. Since it is plausible that we gain the more bits, the more strongly dependent the two attributes are (because in this case a value of one of the attributes leaves fewer choices for the value of the other), we may use the Hartley information gain as a direct indication of the strength of dependence of the two attributes.

The Hartley information gain is generalized to the specificity gain [Gebhardt and Kruse 1996, Borgelt and Kruse 1997a, Borgelt 2000] as shown in figure 2: It is integrated over all $\alpha$-cuts of a given possibility distribution.

$$
\begin{aligned}
S_{\text {gain }}(A, B)=\int_{0}^{\text {sup } \pi} & \log _{2}\left(\sum_{a \in \operatorname{dom}(A)}[\pi]_{\alpha}(A=a)\right) \\
& +\log _{2}\left(\sum_{b \in \operatorname{dom}(B)}[\pi]_{\alpha}(B=b)\right) \\
& -\log _{2}\left(\sum_{a \in \operatorname{dom}(A)} \sum_{b \in \operatorname{dom}(B)}[\pi]_{\alpha}(A=a, B=b)\right) \mathrm{d} \alpha .
\end{aligned}
$$

Surveys of other evaluation measures - which include probabilistic measurescan be found in [Borgelt and Kruse 1997a, Borgelt 2000].

\subsection{Search Methods}

As already indicated above, a search method determines which graphs are considered in order to find a good graphical model. Since an exhaustive search is impossible due to the huge number of graphs (there are $2\left(\begin{array}{l}n \\ 2\end{array}\right)$ possible undirected graphs over $n$ attributes), heuristic search methods have to be used. Usually these heuristic methods introduce strong restrictions w.r.t. the graphs considered and exploit the value of the chosen evaluation measure to guide the search. In addition they are often greedy w.r.t. the model quality. 

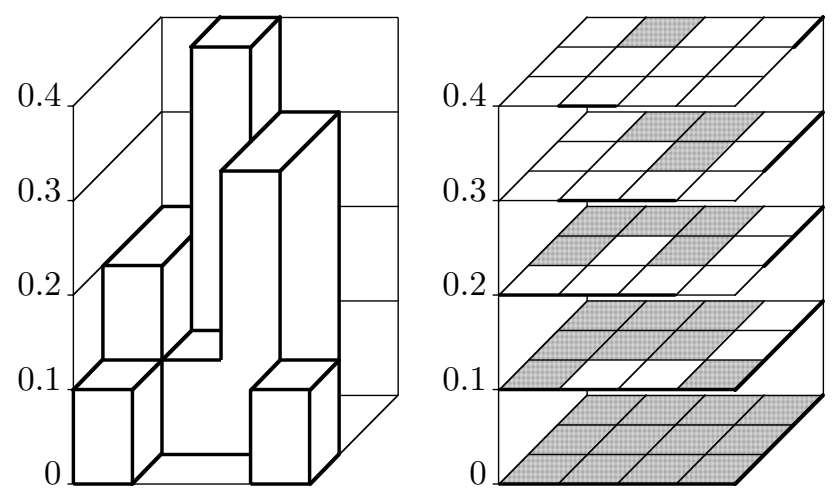

$$
\begin{aligned}
& \log _{2} 1+\log _{2} 1-\log _{2} 1=0 \\
& \log _{2} 2+\log _{2} 2-\log _{2} 3 \approx 0.42 \\
& \log _{2} 3+\log _{2} 2-\log _{2} 5 \approx 0.26 \\
& \log _{2} 4+\log _{2} 3-\log _{2} 8 \approx 0.58 \\
& \log _{2} 4+\log _{2} 3-\log _{2} 12=0
\end{aligned}
$$

Figure 2: Illustration of the idea of specificity gain.

The simplest instance of such a search method is, of course, the Kruskal algorithm [Kruskal 1956], which determines an optimum weight spanning tree for given edge weights. This algorithm has been used very early in the probabilistic setting by [Chow and Liu 1968], who used the Shannon information gain (also called mutual information or cross entropy) of the connected attributes as edge weights. In the possibilistic setting, we may simply replace the Shannon information gain by the specificity gain in order to arrive at an analogous algorithm [Gebhardt and Kruse 1996, Borgelt 2000].

A natural extension of the Kruskal algorithm is a greedy parent selection for directed graphs, which is often carried out on a topological order of the attributes that is fixed in advance ${ }^{1}$ : At the beginning the value of an evaluation measure is computed for a parentless child attribute. Then in turn each of the parent candidates (the attributes preceding the child in the topological order) is temporarily added and the evaluation measure is recomputed. The parent candidate yielding the highest value of the evaluation measure is selected as a first parent and permanently added. In the third step each remaining parent candidate is added temporarily as a second parent and again the evaluation measure is recomputed. As before, the parent candidate that yields the highest value is permanently added. The process stops if either no more parent candidates are available, a given maximal number of parents is reached, or none of the parent candidates, if added, yields a value of the evaluation measure exceeding the best value of the preceding step.

This search method has been used by [Cooper and Herskovits 1992] in the well-known K2 algorithm. As an evaluation measure they used what has become known as the K2 metric. This measure has later been generalized by [Heckerman et al. 1995] to the Bayesian-Dirichlet metric. Of course, in the possibilistic setting we may also apply this search method, again relying on the

\footnotetext{
${ }^{1} \mathrm{~A}$ topological order is an order of the nodes of a graph such that all parent nodes of a given node precede it in the order. That is, there cannot be an edge from a node to a node, which precedes it in the topological order. By fixing a topological order in advance, the set of possible graphs is severely restricted and it is ensured that the resulting graph is acyclic.
} 


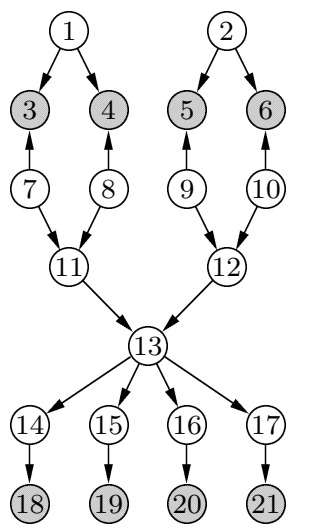

$\begin{array}{ll}21 \text { attributes: } & 11 \text { - offspring phenogroup } 1 \\ 1 \text { - dam correct? } & 12 \text { - offspring phenogroup } 2 \\ 2 \text { - sire correct? } & 13 \text { - offspring genotype } \\ 3 \text { - stated dam phenogroup } 1 & 14 \text { - factor } 40 \\ 4 \text { - stated dam phenogroup } 2 & 15 \text { - factor } 41 \\ 5 \text { - stated sire phenogroup } 1 & 16 \text { - factor } 42 \\ 6 \text { - stated sire phenogroup } 2 & 17 \text { - factor } 43 \\ 7 \text { - true dam phenogroup } 1 & 18 \text { - lysis } 40 \\ 8 \text { - true dam phenogroup } 2 & 19 \text { - lysis } 41 \\ 9 \text { - true sire phenogroup } 1 & 20 \text { - lysis } 42 \\ 10 \text { - true sire phenogroup } 2 & 21 \text { - lysis } 43\end{array}$

The grey nodes correspond to observable attributes.

Figure 3: Domain expert designed network for the Danish Jersey cattle blood type determination example.

specificity gain as the evaluation measure. In order to handle multiple parent attributes with it, we simply combine all parents into one pseudo-attribute and compute the specificity gain for this pseudo-attribute and the child attribute.

\subsection{An Example Application}

As an example of a possible application we consider the problem of blood group determination of Danish Jersey cattle in the F-blood group system [Rasmussen 1992]. For this problem there is a Bayesian network designed by human domain experts, which serves the purpose to verify parentage for pedigree registration.

The section of the world modeled in this example is described by 21 attributes, eight of which are observable. The size of the domains of these attributes ranges from two to eight values. The total frame of discernment has $2^{6} \cdot 3^{10} \cdot 6 \cdot 8^{4}=92876046336$ possible states. This number makes it obvious that the knowledge about this world section must be decomposed in order to make reasoning feasible, since it is clearly impossible to store a probability for each state. Figure 3 lists the attributes and shows the conditional independence graph of the Bayesian network.

As described above, a conditional independence graph enables us to factorize the joint probability distribution into a product of conditional probabilities. In the Danish Jersey cattle example, this factorization leads to a considerable simplification: Only 308 conditional probabilities have to be specified. An example of a conditional probability table, which is part of the factorization, is shown in table 1 . It states the conditional probabilities of the phenogroup 1 of the stated sire of a given calf conditioned on the phenogroup 1 of the true sire and whether the sire was correctly identified. The numbers in this table are derived from statistical data and the experience of human domain experts.

Besides the domain expert designed reference structure there is a database of 500 real world sample cases (an extract of this database is shown in table 2). This database can be used to test learning algorithms for graphical models, 


\begin{tabular}{|c|c||lll|}
\hline sire & true sire & \multicolumn{3}{|c|}{ stated sire ph.gr. 1 } \\
correct & ph.gr. 1 & F1 & V1 & V2 \\
\hline \hline yes & F1 & 1 & 0 & 0 \\
yes & V1 & 0 & 1 & 0 \\
yes & V2 & 0 & 0 & 1 \\
no & F1 & 0.58 & 0.10 & 0.32 \\
no & V1 & 0.58 & 0.10 & 0.32 \\
no & V2 & 0.58 & 0.10 & 0.32 \\
\hline
\end{tabular}

Table 1: An example of a conditional probability distribution that is associated with the conditional independence graph shown in figure 3.

$\begin{array}{lllllllllllllllllllllll}\mathrm{n} & \mathrm{y} & \mathrm{y} & \mathrm{f} 1 & \mathrm{v} 2 & \mathrm{f} 1 & \mathrm{v} 2 & \mathrm{f} 1 & \mathrm{v} 2 & \mathrm{f} 1 & \mathrm{v} 2 & \mathrm{v} 2 & \mathrm{v} 2 & \mathrm{v} 2 \mathrm{v} 2 & \mathrm{n} & \mathrm{y} & \mathrm{n} & \mathrm{y} & 0 & 6 & 0 & 6 \\ \mathrm{n} & \mathrm{y} & \mathrm{y} & \mathrm{f} 1 & \mathrm{v} 2 & * * & * * & \mathrm{f} 1 & \mathrm{v} 2 & * * & * * & * * & * * & \mathrm{f} 1 \mathrm{v} 2 & \mathrm{y} & \mathrm{y} & \mathrm{n} & \mathrm{y} & 7 & 6 & 0 & 7 \\ \mathrm{n} & \mathrm{y} & \mathrm{y} & \mathrm{f} 1 & \mathrm{v} 2 & \mathrm{f} 1 & \mathrm{f} 1 & \mathrm{f} 1 & \mathrm{v} 2 & \mathrm{f} 1 & \mathrm{f} 1 & \mathrm{f} 1 & \mathrm{f} 1 & \mathrm{f} 1 \mathrm{f} 1 & \mathrm{y} & \mathrm{y} & \mathrm{n} & \mathrm{n} & 7 & 7 & 0 & 0 \\ \mathrm{n} & \mathrm{y} & \mathrm{y} & \mathrm{f} 1 & \mathrm{v} 2 & \mathrm{f} 1 & \mathrm{f} 1 & \mathrm{f} 1 & \mathrm{v} 2 & \mathrm{f} 1 & \mathrm{f} 1 & \mathrm{f} 1 & \mathrm{f} 1 & \mathrm{f} 1 \mathrm{f} 1 & \mathrm{y} & \mathrm{y} & \mathrm{n} & \mathrm{n} & 7 & 7 & 0 & 0 \\ \mathrm{n} & \mathrm{y} & \mathrm{y} & \mathrm{f} 1 & \mathrm{v} 2 & \mathrm{f} 1 & \mathrm{v} 1 & \mathrm{f} 1 & \mathrm{v} 2 & \mathrm{f} 1 & \mathrm{v} 1 & \mathrm{v} 2 & \mathrm{f} 1 & \mathrm{f} 1 \mathrm{v} 2 & \mathrm{y} & \mathrm{y} & \mathrm{n} & \mathrm{y} & 7 & 7 & 0 & 7 \\ \mathrm{n} & \mathrm{y} & \mathrm{y} & \mathrm{f} 1 & \mathrm{f} 1 & * * & * * & \mathrm{f} 1 & \mathrm{f} 1 & * * & * * & \mathrm{f} 1 & \mathrm{f} 1 & \mathrm{f} 1 \mathrm{f} 1 & \mathrm{y} & \mathrm{y} & \mathrm{n} & \mathrm{n} & 6 & 6 & 0 & 0 \\ \mathrm{n} & \mathrm{y} & \mathrm{y} & \mathrm{f} 1 & \mathrm{v} 1 & * * & * * & \mathrm{f} 1 & \mathrm{v} 1 & * * & * * & \mathrm{v} 1 & \mathrm{v} 2 & \mathrm{v} 1 \mathrm{v} 2 & \mathrm{n} & \mathrm{y} & \mathrm{y} & \mathrm{y} & 0 & 5 & 4 & 5 \\ \mathrm{n} & \mathrm{y} & \mathrm{y} & \mathrm{f} 1 & \mathrm{v} 2 & \mathrm{f} 1 & \mathrm{v} 1 & \mathrm{f} 1 & \mathrm{v} 2 & \mathrm{f} 1 & \mathrm{v} 1 & \mathrm{f} 1 & \mathrm{v} 1 & \mathrm{f} 1 \mathrm{v} 1 & \mathrm{y} & \mathrm{y} & \mathrm{y} & \mathrm{y} & 7 & 7 & 6 & 7\end{array}$

Table 2: An extract from the Danish Jersey cattle database.

because the quality of the learning result can be determined by comparing it to the reference structure. However, there is a problem connected with the database, namely that it contains a fairly large number of unknown values only a little over half of the tuples are complete (This can already be guessed from the extract shown in table 2: the stars denote missing values).

As already indicated above, missing values make it difficult to learn a Bayesian network, since an unknown value can be seen as representing imprecise information: It states that all values contained in the domain of the corresponding attribute are possible. Nevertheless it is still feasible to learn a Bayesian network from the database in this case, since the dependencies are rather strong and thus the remaining small number of tuples is still sufficient to recover the underlying structure. However, learning a possibilistic network from the same dataset is much easier, since possibility theory was especially designed to handle imprecise information. Hence no discarding or special treatment of tuples with missing values is necessary.

In order to check this conjecture, we implemented the learning methods discussed above (together with their probabilistic counterparts) in a prototype program called INES (Induction of NEtwork Structures). The networks induced with different evaluation measures are very similar to the domain expert designed reference structure, even though the reference structure is a Bayesian network, which may differ from the corresponding possibilistic network, since it employs a different notion of conditional independence. Evaluations of the learned networks showed that their quality is comparable to that of learned probabilistic networks and the reference structure w.r.t. reasoning. 


\section{Conclusion}

In this paper we presented possibilistic graphical models and approaches to learn them from a database of sample cases as an alternative to the betterknown probabilistic approaches. Based on the context model interpretation of a degree of possibility we showed that imprecise data are easily handled in such a possibilistic approach. W.r.t. learning algorithms a lot of work done in the probabilistic counterpart of this research area can be transferred. In general all search methods are directly usable, only the evaluation measures have to be adapted. Experiments done with an example application showed that learning possibilistic networks from data is an important alternative to the established probabilistic methods.

\section{References}

[Andersen et al. 1989] S.K. Andersen, K.G. Olesen, F.V. Jensen, and F. Jensen. HUGIN - A Shell for Building Bayesian Belief Universes for Expert Systems. Proc. 11th Int. J. Conf. on Artificial Intelligence (IJCAI'89, Detroit, MI, USA), 1080-1085. Morgan Kaufmann, San Mateo, CA, USA 1989

[Bauer et al. 1997] E. Bauer, D. Koller, and Y. Singer. Update Rules for Parameter Estimation in Bayesian Networks. Proc. 13th Conf. on Uncertainty in Artificial Intelligence (UAI'97, Providence, RI, USA), 3-13. Morgan Kaufmann, San Mateo, CA, USA 1997

[Borgelt and Kruse 1997a] C. Borgelt and R. Kruse. Evaluation Measures for Learning Probabilistic and Possibilistic Networks. Proc. 6th IEEE Int. Conf. on Fuzzy Systems (FUZZ-IEEE'97, Barcelona, Spain), Vol. 2:10341038. IEEE Press, Piscataway, NJ, USA 1997

[Borgelt and Kruse 1997b] C. Borgelt and R. Kruse. Some Experimental Results on Learning Probabilistic and Possibilistic Networks with Different Evaluation Measures. Proc. 1st Int. J. Conf. on Qualitative and Quantitative Practical Reasoning (ECSQARU/FAPR'9'7, Bad Honnef, Germany), LNAI 1244, 71-85.

[Borgelt and Kruse 1998] C. Borgelt and R. Kruse. Efficient Maximum Projection of Database-Induced Multivariate Possibility Distributions. Proc. 7th IEEE Int. Conf. on Fuzzy Systems (FUZZ-IEEE'98, Anchorage, Alaska, USA), CD-ROM. IEEE Press, Piscataway, NJ, USA 1998

[Borgelt 2000] C. Borgelt. Data Mining with Graphical Models. Ph.D. Thesis, Otto-von-Guericke-University of Magdeburg, Germany 2000

[Castillo et al. 1997] E. Castillo, J.M. Gutierrez, and A.S. Hadi. Expert Systems and Probabilistic Network Models. Springer, New York, NY, USA 1997

[Chickering et al. 1994] D.M. Chickering, D. Geiger, and D. Heckerman. Learning Bayesian Networks is NP-Hard (Technical Report MSR-TR-94- 
17). Microsoft Research, Advanced Technology Division, Redmond, WA, USA 1994

[Chow and Liu 1968] C.K. Chow and C.N. Liu. Approximating Discrete Probability Distributions with Dependence Trees. IEEE Trans. on Information Theory 14(3):462-467. IEEE Press, Piscataway, NJ, USA 1968

[Cooper and Herskovits 1992] G.F. Cooper and E. Herskovits. A Bayesian Method for the Induction of Probabilistic Networks from Data. Machine Learning 9:309-347. Kluwer, Dordrecht, Netherlands 1992

[Dechter and Pearl 1992] R. Dechter and J. Pearl. Structure Identification in Relational Data. Artificial Intelligence 58:237-270. North-Holland, Amsterdam, Netherlands 1992

[Dempster et al. 1977] A.P. Dempster, N. Laird, and D. Rubin. Maximum Likelihood from Incomplete Data via the EM Algorithm. Journal of the Royal Statistical Society (Series B) 39:1-38. Blackwell, Oxford, United Kingdom 1977

[Friedman 1998] N. Friedman. The Bayesian Structural EM Algorithm. Proc. 14 th Conf. on Uncertainty in Artificial Intelligence (UAI'98, Madison, WI, USA), 80-89. Morgan Kaufmann, San Mateo, CA, USA 1997

[Gebhardt and Kruse 1993] J. Gebhardt and R. Kruse. The Context Model - An Integrating View of Vagueness and Uncertainty. Int. Journal of Approximate Reasoning 9:283-314. North-Holland, Amsterdam, Netherlands 1993

[Gebhardt and Kruse 1995] J. Gebhardt and R. Kruse. Learning Possibilistic Networks from Data. Proc. 5th Int. Workshop on Artificial Intelligence and Statistics (Fort Lauderdale, FL, USA), 233-244. Springer, New York, NY, USA 1995

[Gebhardt and Kruse 1996] J. Gebhardt and R. Kruse. Tightest Hypertree Decompositions of Multivariate Possibility Distributions. Proc. 7th Int. Conf. on Information Processing and Management of Uncertainty in Knowledge-based Systems (IPMU'96, Granada, Spain), 923-927. Universidad de Granada, Spain 1996

[Gebhardt 1997] J. Gebhardt. Learning from Data: Possibilistic Graphical Models. Habilitation Thesis, University of Braunschweig, Germany 1997

[Hammersley and Clifford 1971] J.M. Hammersley and P.E. Clifford. Markov Fields on Finite Graphs and Lattices. Unpublished manuscript, 1971. Cited in: [Isham 1981]

[Hartley 1928] R.V.L. Hartley. Transmission of Information. The Bell Systems Technical Journal 7:535-563. Bell Laboratories, USA 1928

[Heckerman 1991] D. Heckerman. Probabilistic Similarity Networks. MIT Press, Cambridge, MA, USA 1991

[Heckerman et al. 1995] D. Heckerman, D. Geiger, and D.M. Chickering. Learning Bayesian Networks: The Combination of Knowledge and Statistical Data. Machine Learning 20:197-243. Kluwer, Dordrecht, Netherlands 1995 
[Isham 1981] V. Isham. An Introduction to Spatial Point Processes and Markov Random Fields. Int. Statistical Review 49:21-43. Int. Statistical Institute, Voorburg, Netherlands 1981

[Jordan 1998] M.I. Jordan, ed. Learning in Graphical Models. MIT Press, Cambridge, MA, USA 1998

[Kruse et al. 1994] R. Kruse, J. Gebhardt, and F. Klawonn. Foundations of Fuzzy Systems, J. Wiley \& Sons, Chichester, United Kingdom 1994.

[Kruskal 1956] J.B. Kruskal. On the Shortest Spanning Subtree of a Graph and the Traveling Salesman Problem. Proc. American Mathematical Society 7(1):48-50. American Mathematical Society, Providence, RI, USA 1956

[Lauritzen and Spiegelhalter 1988] S.L. Lauritzen and D.J. Spiegelhalter. Local Computations with Probabilities on Graphical Structures and Their Application to Expert Systems. Journal of the Royal Statistical Society, Series B, 2(50):157-224. Blackwell, Oxford, United Kingdom 1988

[Lauritzen 1996] S.L. Lauritzen. Graphical Models. Oxford University Press, Oxford, United Kingdom 1996

[Pearl 1988] J. Pearl. Probabilistic Reasoning in Intelligent Systems: Networks of Plausible Inference. Morgan Kaufmann, San Mateo, CA, USA 1988 (2nd edition 1992)

[Rasmussen 1992] L.K. Rasmussen. Blood Group Determination of Danish Jersey Cattle in the F-blood Group System (Dina Research Report 8). Dina Foulum, Tjele, Denmark 1992

[Saffiotti and Umkehrer 1991] A. Saffiotti and E. Umkehrer. PULCINELLA: A General Tool for Propagating Uncertainty in Valuation Networks. Proc. 7th Conf. on Uncertainty in Artificial Intelligence (UAI'91, Los Angeles, CA, USA), 323-331. Morgan Kaufmann, San Mateo, CA, USA 1991

[Shachter et al. 1990] R.D. Shachter, T.S. Levitt, L.N. Kanal, and J.F. Lemmer, eds. Uncertainty in Artificial Intelligence 4. North Holland, Amsterdam, Netherlands 1990

[Shenoy 1992] P.P. Shenoy. Valuation-based Systems: A Framework for Managing Uncertainty in Expert Systems. In: [Zadeh and Kacprzyk 1992], 83-104

[Ullman 1988] J.D. Ullman. Principles of Database and Knowledge-Base Systems, Vol. 1 \& 2. Computer Science Press, Rockville, MD, USA 1988

[Verma and Pearl 1990] T.S. Verma and J. Pearl. Causal Networks: Semantics and Expressiveness. In: [Shachter et al. 1990], 69-76

[Zadeh and Kacprzyk 1992] L.A. Zadeh and J. Kacprzyk. Fuzzy Logic for the Management of Uncertainty. J. Wiley \& Sons, New York, NY, USA 1992 